Article

\title{
Low Adenovirus Vaccine Doses Administered to Skin Using Microneedle Patches Induce Better Functional Antibody Immunogenicity as Compared to Systemic Injection
}

\author{
Olivia Flynn ${ }^{1}$, Kate Dillane ${ }^{1}$, Juliane Sousa Lanza ${ }^{1}$, Jennifer M. Marshall ${ }^{2}$, Jing Jin ${ }^{2}$, Sarah E. Silk ${ }^{2}$, \\ Simon J. Draper ${ }^{2} \mathbb{D}$ and Anne C. Moore ${ }^{1,3, * \mathbb{D}}$ \\ 1 School of Pharmacy, University College Cork, T12 XF62 Cork, Ireland; olivia.flynn@cit.ie (O.F.); \\ kdillane@ucc.ie (K.D.); julianelanza@gmail.com (J.S.L.) \\ 2 The Jenner Institute, University of Oxford, Oxford OX3 7DQ, UK; jennifer.marshall@ndm.ox.ac.uk (J.M.M.); \\ jing.jin@ndm.ox.ac.uk (J.J.); sarah.silk@ndm.ox.ac.uk (S.E.S.); simon.draper@ndm.ox.ac.uk (S.J.D.) \\ 3 School of Biochemistry and Cell Biology, University College Cork, T12 XF62 Cork, Ireland \\ * Correspondence: anne.moore@ucc.ie
}

Citation: Flynn, O.; Dillane, K.; Lanza, J.S.; Marshall, J.M.; Jin, J.; Silk, S.E.; Draper, S.J.; Moore, A.C. Low Adenovirus Vaccine Doses Administered to Skin Using Microneedle Patches Induce Better Functional Antibody Immunogenicity as Compared to Systemic Injection. Vaccines 2021, 9, 299. https:// doi.org/10.3390/vaccines9030299

Academic Editor: Vasso

Apostolopoulos

Received: 20 January 2021

Accepted: 27 February 2021

Published: 22 March 2021

Publisher's Note: MDPI stays neutra with regard to jurisdictional claims in published maps and institutional affiliations.

Copyright: (c) 2021 by the authors. Licensee MDPI, Basel, Switzerland. This article is an open access article distributed under the terms and conditions of the Creative Commons Attribution (CC BY) license (https:// creativecommons.org/licenses/by/ $4.0 /)$.
Abstract: Adenovirus-based vaccines are demonstrating promising clinical potential for multiple infectious diseases, including COVID-19. However, the immunogenicity of the vector itself decreases its effectiveness as a boosting vaccine due to the induction of strong anti-vector neutralizing immunity. Here we determined how dissolvable microneedle patches (DMN) for skin immunization can overcome this issue, using a clinically-relevant adenovirus-based Plasmodium falciparum malaria vaccine, AdHu5-PfRH5, in mice. Incorporation of vaccine into patches significantly enhanced its thermostability compared to the liquid form. Conventional high dose repeated immunization by the intramuscular (IM) route induced low antigen-specific IgG titres and high anti-vector immunity. A low priming dose of vaccine, by the IM route, but more so using DMN patches, induced the most efficacious immune responses, assessed by parasite growth inhibitory activity (GIA) assays. Administration of low dose AdHu5-PfRH5 using patches to the skin, boosted by high dose IM, induced the highest antigen-specific serum IgG response after boosting, the greatest skewing of the antibody response towards the antigen and away from the vector, and the highest efficacy. This study therefore demonstrates that repeated use of the same adenovirus vaccine can be highly immunogenic towards the transgene if a low dose is used to prime the response. It also provides a method of stabilizing adenovirus vaccine, in easy-to-administer dissolvable microneedle patches, permitting storage and distribution out of cold chain.

Keywords: adenovirus; virus vector vaccine; microneedle; skin; dose; stability; antibody; antivector immunity

\section{Introduction}

The high cost of maintaining vaccine cold chains has been identified as a key challenge that must be overcome to achieve global immunization goals. Live and whole inactivated viruses remain a sizeable proportion of clinically used vaccines. Recombinant virus vectors, and in particular, replication incompetent, recombinant adenoviruses are leading vaccine candidates for old, emerging, and pandemic diseases, including several candidates to protect against SARS-CoV-2-induced COVID-19 [1-3]. These vaccines are heat sensitive and must be stored and distributed at low temperatures. Stabilization of live virus vaccines is challenging, but overcoming this issue is a critical factor for the successful and equitable deployment of vaccines in the field [4].

Induction of immunity to the adenovirus vector severely limits its repeated use due to immune-mediated neutralization of the re-administered virus. Clinically, this is being addressed through the use of heterologous prime-boost immunization, where a 
different vaccine platform, such as the poxvirus vector MVA (Modified Vaccinia Virus Ankara) [5-8] or subunit, adjuvanted protein [9] is used in the second and subsequent immunizations, or through the use of serologically different adenoviruses [10,11]. Although strong antigen-specific immunity can be induced in clinical trials, the costs and logistics of manufacturing and distributing two or more vaccines and administering them in the correct sequence in every individual, would need to be economically justified before widespread implementation, or alternatively only utilized in epidemic outbreaks.

We previously demonstrated that adenovirus vaccine administration using silicon microneedles permitted repeated use of the same adenovirus vaccine due to the induction of significantly lower anti-vector antibody responses compared to injection with a needle and syringe in mice [12]. However, although silicon microneedles overcome some logistic issues, they do not address vaccine stability. Dissolvable microneedles are micron-scale protrusions that are sharp enough to insert into the skin. They are composed of suitable excipients that incorporate, and should stabilize, vaccines in a dry, solid state. On skin insertion the microneedle material dissolves and the vaccine is delivered to the body. Dissolvable microneedle (DMN) patches for skin-based immunization offer several logistic and economic benefits to healthcare systems and individual end-users, including: ease of use; lack of requirement for vaccine reconstitution; more efficient and cost-effective logistics, by removal of cold chain; the elimination of needles, syringes, and hazardous waste; and small package size. We previously developed a novel, drop-dispensing based DMN patch production method that overcomes the waste associated with conventional methods of dissolvable microneedle production [13-15]. We also developed suitable formulations for stabilizing vaccines in DMN patches to preserve vaccine integrity during microneedle fabrication, upon drying and during subsequent storage [16].

Dose sparing has been suggested as a key benefit of microneedle-mediated vaccine administration, however whether this translates to humans or larger animals has yet to be determined. A small number of published clinical studies have assessed the impact of adenovirus-based vaccine dose on $\mathrm{T}$ cell responses $[17,18]$ and antibody titers [19-21] using conventional intramuscular delivery. We therefore wanted to determine how adenovirus vaccine dose impacts on the induction of antibody responses when the vaccine is administered by the intramuscular (IM) or patch-mediated skin route. Here, we chose human adenovirus serotype 5 (AdHu5) as a model adenovirus without intellectual property restrictions, and which is also suitable for thermostability and administration route studies. The immunogenicity and efficacy of AdHu 5 vaccines can be readily matched by clinically-applicable simian adenoviruses, as previously demonstrated [22,23].

To date, the administration of adenovirus vaccines by microneedle patches in mice has focused on the induction of T cell responses to HIV antigens [24,25], and there has been no understanding of the effect of anti-vector immunity, or the induction of functional/protective humoral immunity using DMN patches in any model. In this study, we address this problem and examine the induction of vector-specific and functional antigenspecific antibody responses using DMN patches fabricated using our simple process.

The development of highly effective and durable vaccines against the human malaria parasites Plasmodium falciparum and P. vivax remains challenging $[5,26]$. One vaccine strategy is targeted at the blood-stage of the disease, by blocking merozoite invasion of red blood cells. This approach will likely require the maintenance of high titres of neutralizing antibodies to merozoite proteins. Plasmodium falciparum reticulocyte-binding protein homolog 5 (RH5) has emerged as a strong blood-stage vaccine candidate, and is being clinically tested. In parallel with this development, non-human primate studies have demonstrated that the in vitro assay of antibody-mediated growth inhibition activity (GIA) against $P$. falciparum serves as an in vitro correlate of vaccine-induced in vivo protection when using vaccines targeting PfRH5 and other blood-stage antigens [27-29].

The objective of this study was therefore to determine how the dose and method of delivery, either IM injection or skin administration using a DMN patch, of an adenovirus 
vectored blood-stage malaria vaccine impacts on the induction of neutralizing anti-parasitic antibodies and anti-vector antibodies in mice.

\section{Methods}

\subsection{Vaccine}

Replication-deficient adenovirus human serotype 5 (AdHu5) expressing full-length Plasmodium falciparum RH5 (AdHu5-PfRH5) was previously described [27,30]. Antigens were cloned into a replication-deficient $\mathrm{AdHu} 5$ genome downstream of a mammalian secretory signal (from human tissue plasminogen activator), and viruses were prepared using previously described techniques for AdHu5. All doses of vaccine are expressed as live, infectious units (ifu) but where relevant in the text, the dose measured in viral particles (vp) is also reported.

\subsection{Dissolvable Microneedle Fabrication}

Pyramidal-shaped silicon microneedles consisting of $5 \times 5$ array (length: $500 \mu \mathrm{m}$, base diameter: $333 \mu \mathrm{m}$, Tyndall National Institute) were used as a molding template for fabrication of microneedle cavities in polydimethylsiloxane (PDMS) molds, as previously described $[15,31,32]$. The vaccine was formulated with a $15 \%(v / v)$ trehalose-based solution for the production of dissolvable microneedle patches. Approximately $7 \mu \mathrm{L}$ of formulation was delivered directly into 25 microneedle pores of a $1 \mathrm{~cm}$ [2] mold. Following delivery of the formulation onto molds, microneedles were dried for $18 \mathrm{~h}$ at room temperature (approximately $21^{\circ} \mathrm{C}$ ) in the presence of desiccant. After transfer onto medical grade adhesive tape (1525 L Poly Med tape, $3 \mathrm{M}$ ) arrays were kept in a sealed container in a dry ambient environment in the presence of desiccant until use.

\subsection{Stability Study}

DMN patches containing AdHu5-PfRH5 with a dose of $1 \times 10^{7}$ ifu per $1 \mathrm{~cm}^{2}$ patch were sealed into nitrogen-purged individual glass vials with desiccant. Packaged and sealed vaccine-loaded DMN were stored in a stability chamber at $25^{\circ} \mathrm{C}$ and $60 \%$ relative humidity (RH), or $40{ }^{\circ} \mathrm{C}$ and $75 \% \mathrm{RH}$, for the indicated time, according to ICH Q1A guidelines [33] ( $n=6$ per group per timepoint, except at 6 months when $n=4$ per group). Samples were dissolved in $32 \mu \mathrm{L}$ complete RPMI media, and viable AdHu5-PfRH5 was quantified using a hexon immunoassay (QuickTiter Immunoassay, Cambridge Bioscience, Cambridge, UK).

\subsection{Immunization}

Female Balb/c mice, 4-6 weeks old (Harlan, Hillcrest, UK), were used in all experiments which were conducted in accordance with the terms of licenses from the Irish Department of Health and Children under the Cruelty to Animals Act (license number B100/4034) and according to the approval of the UCC AECC Committee. Mice were immunized with the dose of vaccine indicated in the text and figure legends. For each patch administration, mice were pre-anesthetized with ketamine hydrochloride and medetomidine hydrochloride, and the patches were applied to the dorsal surface of each ear, pressing the fingers against the animals' skin for $10 \mathrm{~s}$. The ear was then loosely wrapped with Elastoplast to permit the maintenance of the patches on the skin. An anesthetic reversal agent, atipamezole (anti-sedan), was then administered to mice. Patches were in place overnight as previously outlined [34]. Alternatively, vaccine was administered with a conventional $28 \mathrm{G}$ needle and syringe intramuscularly (IM) into the anterior thigh of the hind leg ( $50 \mu \mathrm{L}$ per mouse divided across both legs). Mice were primed on day 0 and boosted by the IM route or using a microneedle patch at day 54 , as outlined in the figures and text. 


\subsection{Immunogenicity Analysis}

Antibody responses to the vector and to the antigen were determined by ELISA as previously described [12,35,36], using $100 \mathrm{ng} /$ well of purified recombinant PfRH5 antigen (called "RH5.1") produced from a Drosophila melanogaster Schneider-2 (S2) stable cell line [37] for antigen-specific responses and AdHu5-mCherry $\left(3 \times 10^{8} \mathrm{vp} /\right.$ well in PBS) for vector-specific responses [12]. Endpoint titres were taken as the $x$-axis intercept of the dilution curve at an absorbance value $3 \times$ standard deviations greater than the $\mathrm{OD}_{595}$ for naïve mouse serum (typical cut-off $\mathrm{OD}_{595}$ for positive sera $=0.1$ ). A standard serum sample was included in all assays as a reference control.

\subsection{Functional Antibody Efficacy Analysis: Growth Inhibitory Activity Assay}

The ability of antibodies to inhibit growth of P. falciparum 3D7 clone parasites in vitro was assessed by a standardized GIA assay using purified IgG at $1 \mathrm{mg} / \mathrm{mL}$, as previously described in detail $[38,39]$. Briefly, purified IgGs from serum samples were pre-adsorbed with uninfected human $\mathrm{O}+$ erythrocytes to remove any anti-human erythrocyte immunoglobulins, sterile filtered, and heat inactivated. The GIA assay was performed on these samples using human erythrocytes parasitized with late trophozoite and schizont stages of $P$. falciparum. Parasite growth after $40 \mathrm{~h}$ of culture was determined by a biochemical assay specific for parasite lactate dehydrogenase, and the results were determined by the OD650. Values obtained with test IgGs were compared with those obtained with parasites incubated with a pool of malaria-naïve human control serum and with uninfected red cells, and are reported as \% GIA in the test sample versus control.

\section{Results}

\subsection{Long-Term Vaccine Stabilisation Outside Cold Chain Conditions}

We previously demonstrated the short-term stability of an AdHu5 vector embedded in our DMN patches and kept at ambient temperature over 14 days [15]. Here, we stressed the vaccine at ambient and high temperature conditions for 6 months to better determine the vaccine's stability in dissolvable microneedle patches. AdHu5-PfRH5 vaccine-containing DMN patches were stored at $25{ }^{\circ} \mathrm{C}$ and $60 \%$ relative humidity $(\mathrm{RH})$, or $40{ }^{\circ} \mathrm{C}$ and $75 \% \mathrm{RH}$, for up to 6 months, according to ICH Q1A guidelines [33]. Adenovirus infectivity was assessed in patches or in liquid at 1 week or 1, 2, 3, and 6 months. Adenovirus stored in the liquid form at the higher temperature, quickly degraded within a week, and viable virus was undetectable at one month (Figure 1A). In contrast, no degradation of the vaccine in patches was observed at $40^{\circ} \mathrm{C}$ for up to 2 months, however vaccine degradation was observed when patches were stored at $40{ }^{\circ} \mathrm{C}$ for 3 months. No visible changes to the microneedles were observed in these patches stored at $40^{\circ} \mathrm{C}$ with up to 6 months storage (Figure 1C). At the accelerated temperature of $25^{\circ} \mathrm{C}$, adenovirus vaccine incorporated into patches was fully stabilized; no loss in viable vaccine (Figure 1A) or visible changes (Figure 1B) could be detected at 6 months. These results demonstrate the significant improvement to the stability of this adenovirus vaccine by incorporation into patches.

3.2. Low Dose Skin Administration Significantly Enhances Antigen-Specific Antibody Responses Induced by Repeated Adenovirus Vaccination

We previously demonstrated influenza vaccine dose sparing using dissolvable microneedles in mice [15]. As adenovirus is a live vaccine where one virus unit can produce an unknown antigen dose in situ, we aimed to determine if dose sparing would be relevant to adenovirus vaccines. To address this question, a range of doses of adenovirus expressing P. falciparum RH5 antigen, AdHu5-PfRH5 [30], was administered by patches or in liquid format by the IM route to Balb/c mice (Supplementary Figure S1). A $1 \times 10^{8}$ infectious units, ifu $\left(1.6 \times 10^{9}\right.$ virus particles, vp) vaccine dose by the IM route represented the dose and route of immunization conventionally administered to mice [40]. Only the lowest doses of vaccine by the IM or patch route induced a significantly lower IgG response to the PfRH5 antigen, compared to the conventional vaccine dose $\left(1 \times 10^{8}\right.$ ifu $), 8$ weeks after the primary 
immunization (Figure 2A; light grey bars). With respect to the induction of anti-vector immunity, lower vaccine doses by either IM $\left(\leq 1 \times 10^{5} \mathrm{ifu}\right)$ or skin $\left(\leq 5 \times 10^{5} \mathrm{ifu}\right)$ route, also induced significantly lower anti-adenovirus $\operatorname{IgG}$ responses after the first immunization, compared to the standard $1 \times 10^{8}$ ifu IM immunization (Figure 2B).

(A)

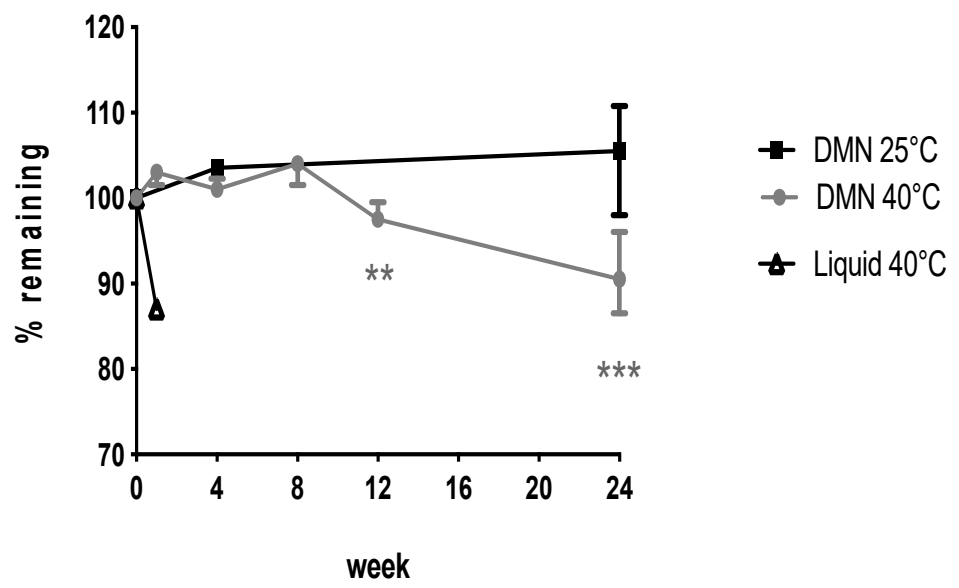

(B)
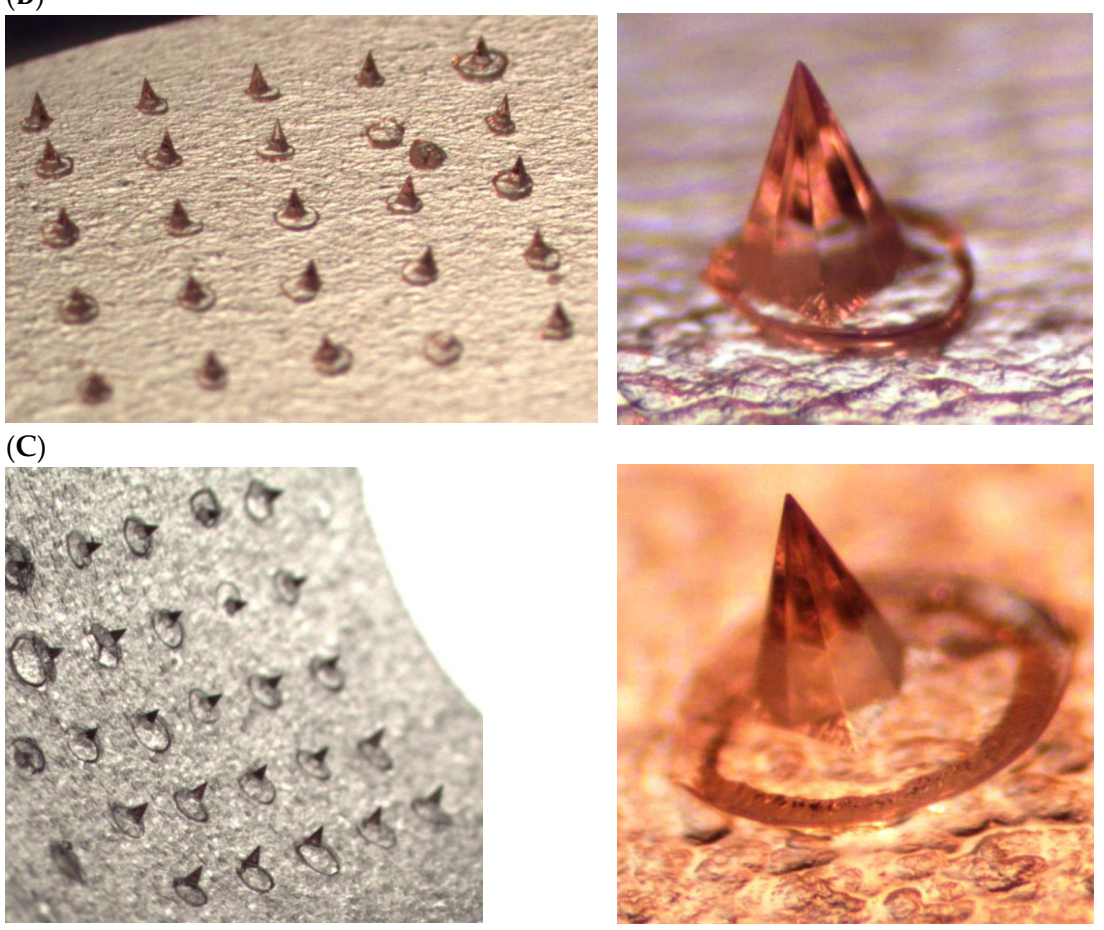

Figure 1. Incorporation of adenovirus vaccine into dissolvable microneedle (DMN) patches significantly stabilized the vaccine for up to 6 months. AdHu5-PfRH5 containing patches were stored in a stability chamber at $25^{\circ} \mathrm{C}$ or $40^{\circ} \mathrm{C}$, with the surrounding chamber maintained at $60 \%$ or $75 \%$ relative humidity (RH), for the indicated time. (A) The initial vaccine dose was $1 \times 10^{7}$ ifu per $1 \mathrm{~cm}^{2}$ patch. The amount of remaining viable adenovirus was measured at 1 week and then 1, 2, 3, and 6 months after incubation of samples ( $n=6$ per group per timepoint, except at 6 months when $n=4$ per group). The $\%$ remaining in the sample was determined compared to time zero. No physical changes were observed in DMN stored at accelerated stability conditions. Points represent median, error bars represent inter-quartile range. ${ }^{* *} p<0.01,{ }^{* * *} p<0.001$, compared to time zero, Kruskal-Wallis, one-way ANOVA, with Dunn's multiple comparison test. (B) $25^{\circ} \mathrm{C}, 60 \% \mathrm{RH}$, or (C) $40{ }^{\circ} \mathrm{C}, 75 \% \mathrm{RH}$, for 6 months. 
A
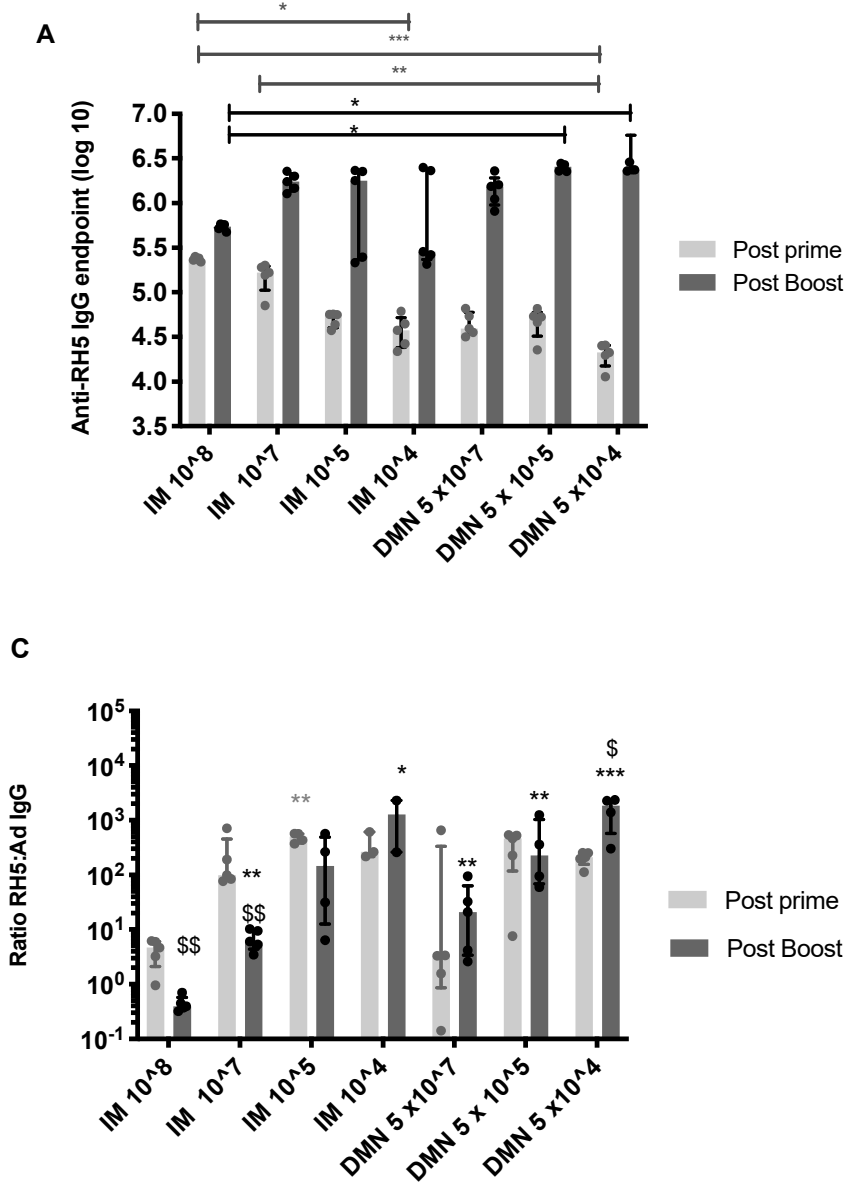

B

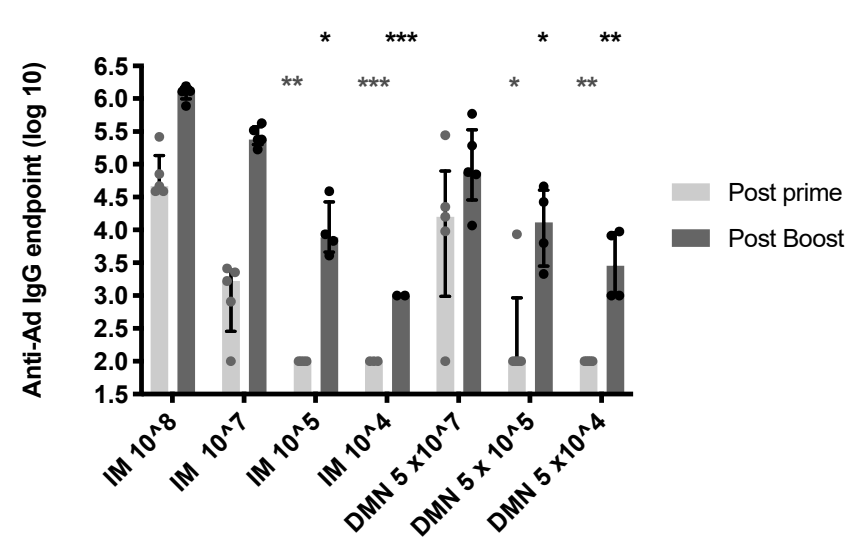

D

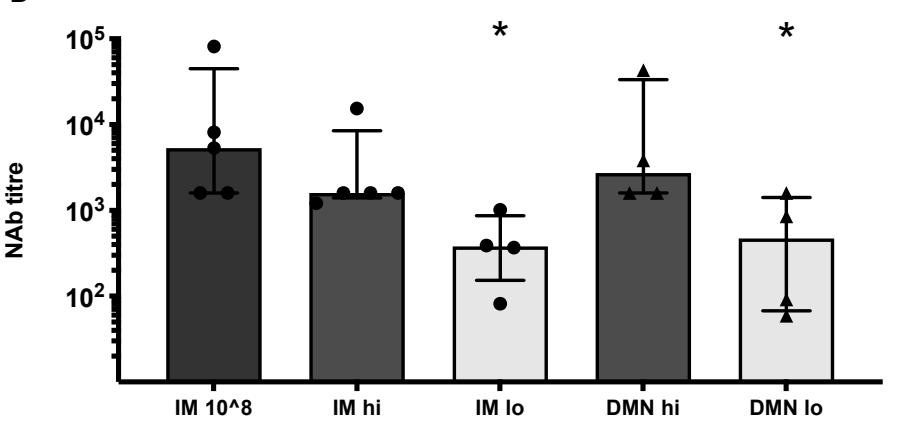

Figure 2. Effect of vaccine dose and route on the induction of IgG antibodies to PfRH5 antigen and to the adenovirus vector. Female Balb/c mice ( $n=5$ per group) were immunized on day 0 with AdHu5-PfRH5 by the IM route or using DMN patches. The following doses were administered by IM (from left to right); $1 \times 10^{8}$ ifu, $1 \times 10^{7}$ ifu, $1 \times 10^{5}$ ifu, or $1 \times 10^{4}$ ifu to each mouse. Alternatively, $5 \times 10^{7}$ ifu, $5 \times 10^{5}$ ifu, or $5 \times 10^{4}$ ifu were administered in two patches to each mouse; one patch per ear. Serum IgG responses were assessed 8 weeks after the priming immunization (light grey bars). All mice were boosted on day 54 with $1 \times 10^{8}$ ifu AdHu5-PfRH5 by the IM route and post-boost IgG responses were assessed 2 weeks after this boost (dark grey bars). (A) IgG responses to the encoded antigen PfRH5. ${ }^{*} p<0.05,{ }^{* *} p<0.01,{ }^{* * *} p<0.001$ by one-way ANOVA, Kruskal-Wallis test with Dunn's multiple comparison. Light grey lines and stars indicate significant differences across post-prime samples, dark grey lines indicate significant differences across post-boost samples. (B) IgG responses to the AdHu5 vector. ${ }^{*} p<0.05,{ }^{* *} p<0.01,{ }^{* * *} p<0.001$ compared to control, IM $1 \times 10^{8}$ ifu. (C) The ratio of anti-PfRH5 IgG titre to anti-vector IgG in each animal. ${ }^{*} p<0.05,{ }^{* *} p<0.01,{ }^{* * *} p<0.001$ compared to control, IM $1 \times 10^{8}$ ifu. $\$ p<0.05, \$ \$ p<0.01$ of post-prime compared to post-boost within the same group. (D) Neutralizing antibody responses to AdHu5 vector 2 weeks after boosting. "IM lo" and "DMN lo" refers to priming mice with a low dose of $1-5 \times 10^{4}$ ifu by IM injection or DMN patch, whereas "IM hi" and "DMN hi" refer to a high dose of 1-5 × 107 ifu by IM or DMN patch-based administration in the prime ${ }^{*} p<0.05$, compared to control, IM $1 \times 10^{8}$ ifu. Bars represent median, error bars represent interquartile range, dots represent individual samples.

All groups were boosted by the same IM route and dose, so that post-boost immunity only reflected differences induced in the primary immunization. All groups had a significantly higher IgG to PfRH5 post-boost, compared to the primary immunization $(p<0.05$, paired $t$-test) (Figure 2A). Surprisingly, mice primed with lower doses of vaccine $\left(5 \times 10^{5}\right.$ ifu and $5 \times 10^{4} \mathrm{ifu}$ ) using DMN patches, but not by the IM route, induced consistent and significantly higher antigen-specific IgG responses compared to the conventional IM immunization (Figure 2A). In contrast, a high level of variability was observed in animals primed with low doses of vaccine by the IM route. The IM high dose boost caused a significant increase in anti-vector IgG in all groups compared to the post-prime anti-vector response 
(Figure 2B). A dose response effect was observed with respect to anti-vector responses post-boost (Figure 2B). Priming doses less than $1 \times 10^{7}$ ifu by either route resulted in significantly lower post-boost anti-vector responses compared to the $1 \times 10^{8}$ ifu IM group.

To determine how anti-AdHu5 IgG antibodies reflected functional, adenovirus neutralizing capacity, titres of neutralizing antibodies (NAb) were determined by hexon infectivity assay for high and low vaccine doses by the IM and skin routes after boosting (Figure 2D). A dose dependence was observed, irrespective of route, whereby low vaccine doses $\left(1-5 \times 10^{4} \mathrm{ifu}\right)$ induced significantly weaker anti-adenovirus NAb titres as compared to priming with $1-5 \times 10^{7}$ ifu or with $1 \times 10^{8}$ ifu IM.

Using the antibody titres to the antigen (Figure 2A) and to the vector (Figure 2B), we analyzed the skewing of the antibody response to the encoded antigen, and away from the virus vector. Conventional immunization, using $1 \times 10^{8}$ ifu AdHu5-PfRH5 by the IM route primed a humoral response, whereby the anti-transgene response was 3.5 times higher than the anti-vector antibody response, as evidenced by an equivalent or higher relative level of vector-specific antibodies compared to antigen-specific antibodies (geometric mean; 3.5, 95\% CI; 1.5 to 9.2) (Figure 2C). Lowering the priming vaccine dose resulted in increased ratios of anti-PfRH5 to anti-vector responses, irrespective of route, compared to a single, conventional IM immunization. Boosting mice that were primed with $1 \times 10^{8} \mathrm{ifu}$ or $1 \times 10^{7} \mathrm{ifu}$ of vaccine by the IM route pushed the ratio of antibodies significantly further towards an anti-vector response, as evidenced by significant decreases in the ratio of anti-PfRH5 antibodies to anti-vector post-boost compared to post-prime. For example, in the IM $1 \times 10^{7}$ ifu group, the post-prime geometric mean ratio was 154 (95\% CI: 49 to 488 ) and post-boost this dropped to a geometric mean ratio of 6 (95\% CI: 3 to 11). There was no significant change in the ratio of antigen- to vector-specific antibodies from prime to boost in the lower doses $\left(1 \times 10^{5}\right.$ or $\left.1 \times 10^{4} \mathrm{ifu}\right) \mathrm{IM}$ groups. Therefore at lower doses given IM, the ratio of antigen to vector specific antibodies could not be modulated by the boost. In contrast, at the lowest vaccine dose administered by DMN patches, the ratio of antigen to vector specific responses was significantly altered, and increased, from prime to boost; the geometric mean ratio post-prime was 198 (95\% CI: 131 to 299) and post-boost this significantly increased to a geometric mean ratio of 1232 (95\% CI: 266 to 5713). This regimen of the lowest dose administered with a patch (DMN $5 \times 10^{4} \mathrm{ifu}$ ) and boosted with a vaccine dose of $1 \times 10^{8}$ ifu by the IM route induced the highest ratio of antigen-specific to vector-specific antibodies; these were significantly higher than the IM $1 \times 10^{8}$ ifu approach, and this was the only prime-boost approach that led to a significant increase in this ratio post-boost. Overall, these results demonstrate that repeated IM immunization favors the expansion of anti-vector immune responses, whereas priming with adenovirus vaccine in DMN patches favors the enrichment of antigen-specific IgG. Priming with low vaccine doses, particularly with a DMN patch, thus favors a further skew towards antigen-specific immunity.

\subsection{Homologous Dose and Route in the Boosting Immunization Can Enhance Antigen-Specific Antibody Responses}

The simplest vaccination regime would be to re-administer the same vaccine dose using the same route in second and subsequent immunizations. However this must be balanced by the induction of strong immunity that provides protection against pathogen challenge. Mice were thus immunized twice with a low $\left(5 \times 10^{4} \mathrm{ifu}\right)$ or high dose $\left(5 \times 10^{7} \mathrm{ifu}\right)$ of AdHu5-PfRH5 by the IM route or with a patch (Figure 3). After the primary immunization, the antibody responses to PfRH5 and to the adenovirus vector demonstrated the same responses as described above. After boosting, mice primed with low dose patches, and boosted with high or low doses patches (DMNlo/DMNlo and DMNlo/DMNhi) had equivalent post-boost anti-PfRH5 antibody responses to repeated high dose IM immunization (Figure 3A). Only high doses of vaccine, by either route, had anti-vector antibodies (Figure 3B). Both of the low dose DMN groups exhibited significantly higher ratios of anti-PfRH5 to anti-vector antibodies after the first immunization, compared to high dose IM immunization $(p<0.05)$ (Figure 3C). After the boost, all groups, including the group 
receiving two high doses in a patch, exhibited significantly higher ratios of anti-PfRH5 to anti-vector antibodies compared to repeated high dose IM immunization. This highlights that mice primed with low vaccine doses had post-boost responses dominated by anti-PfRH5 antibodies, as relative to anti-vector antibodies.

A

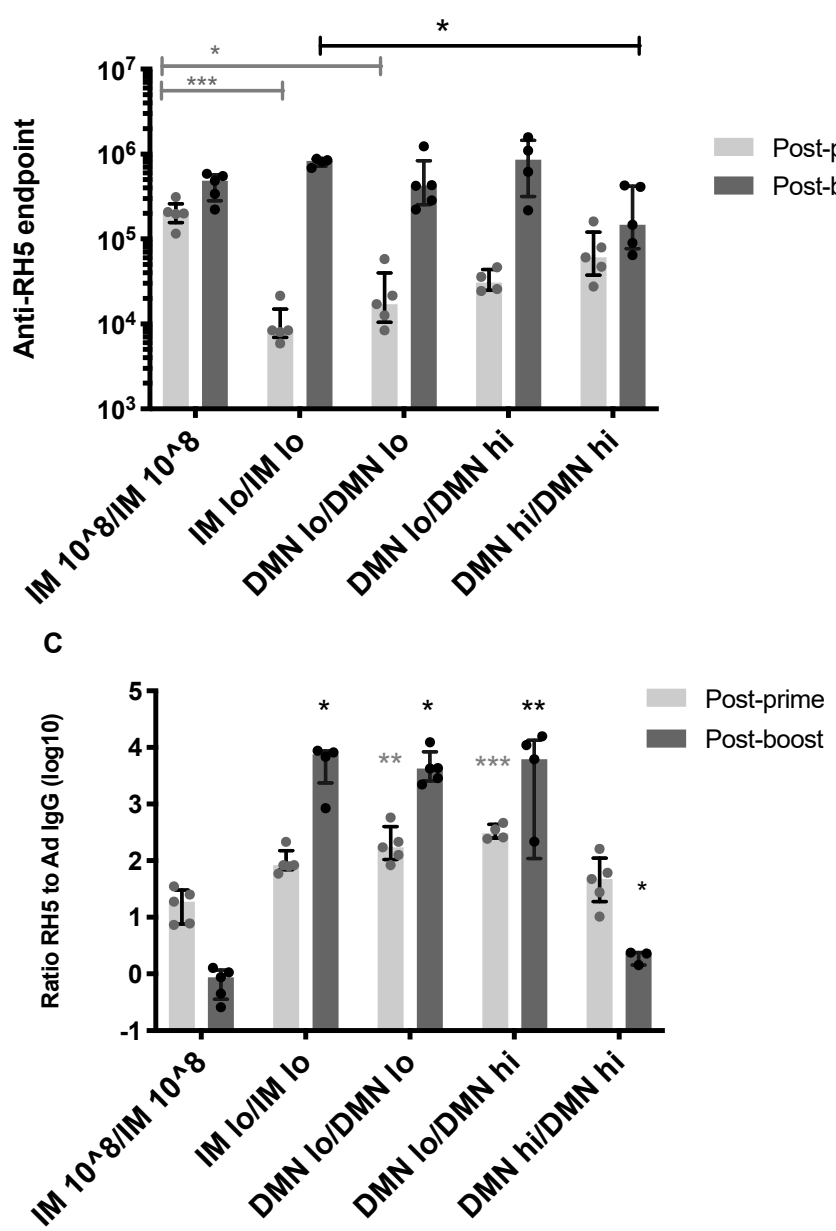

B

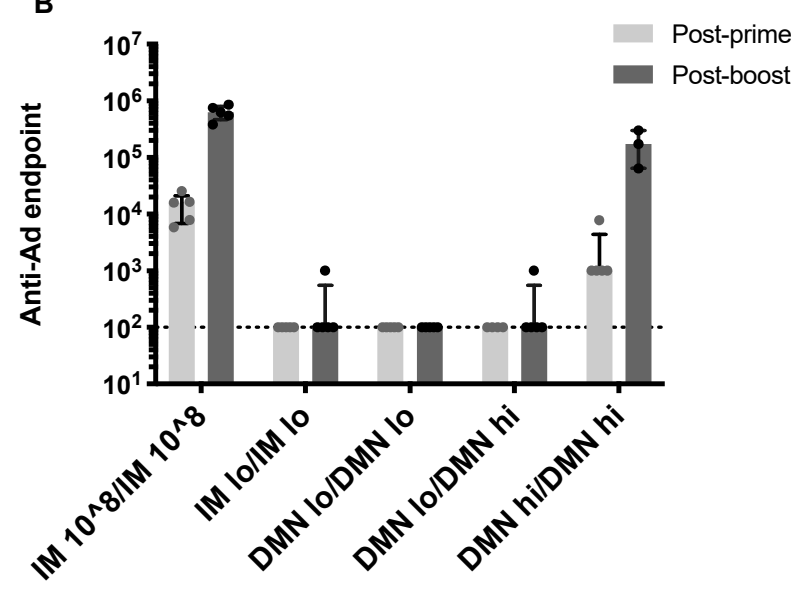

Figure 3. Antibody responses induced using a homologous dose and route in the prime and the boost. Female Balb/c mice ( $n=5$ per group) were immunized on day 0 with AdHu5-PfRh5 by the IM route or using DMN patches. The conventional priming and boosting by the IM route, using $1 \times 10^{8}$ ifu acting as a control. Alternatively, mice were administered a low dose of $5 \times \mathbf{1 0}$ ifu by IM injection or DMN patch ("IM lo" or "DMN lo") or a high dose of $5 \times 10^{7}$ ifu by DMN patch ("DMN hi"). Serum IgG responses were assessed 8 weeks after the priming immunization. All mice were boosted on day 54 with a low or high dose of vaccine by the indicated route, and post-boost IgG responses were assessed 2 weeks after this boost. (A) IgG responses to the encoded antigen PfRH5. (B) IgG responses to the AdHu5 vector. (C) The ratio of the IgG titre to PfRH5 to the vector in each animal. ${ }^{*} p<0.05,{ }^{* *} p<0.01,{ }^{* *} p<0.001$ by one-way ANOVA, Kruskal-Wallis test, with Dunn's multiple comparison test compared to IM 10^8 in all graphs. Bars represent median, error bars represent interquartile range, circles represent individual samples.

These results demonstrate that priming and boosting with a low dose $\left(5 \times 10^{4}\right.$ ifu $)$ of vaccine, using patches or by the IM route, significantly skews the antibody response towards the antigen and away from the vector, compared to high dose administration.

\subsection{Low Dose Priming Induces Higher Levels of Functional Antibodies}

We tested the ability of IgG purified from serum to neutralize P. falciparum parasites, clone 3D7, in a GIA assay [38,39]. In the first study, serum from mice boosted with high dose vaccine by the IM route $\left(1 \times 10^{8} \mathrm{ifu}\right.$; “IM 10^8"), were evaluated (Figure 4A). Repeated high dose IM immunization induced an antibody response that contained low levels of 
growth inhibitory antibodies (median 45\%, 95\% CI; 37\% to 56\%). A high dose of vaccine administered by patch in the prime and boosted with high dose IM (DMN hi/IM 10 ${ }^{8}$ ) did not significantly increase this response. In contrast, priming with a low dose of vaccine, by the IM route or DMN patches and boosting with $1 \times 10^{8}$ ifu IM induced high levels of antibodies in serum that inhibited parasite growth. GIA levels in the patch primed group (median, 83\%, 95\% CI: 80-85\%) were significantly higher compared to the control, IM, immunized group.

A

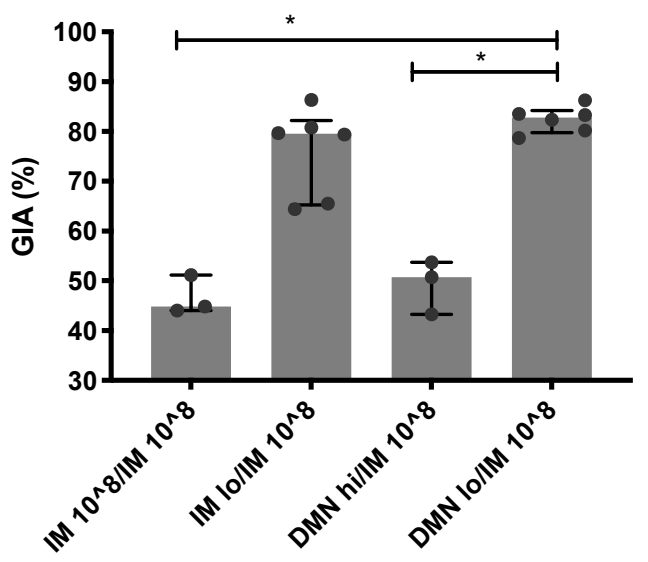

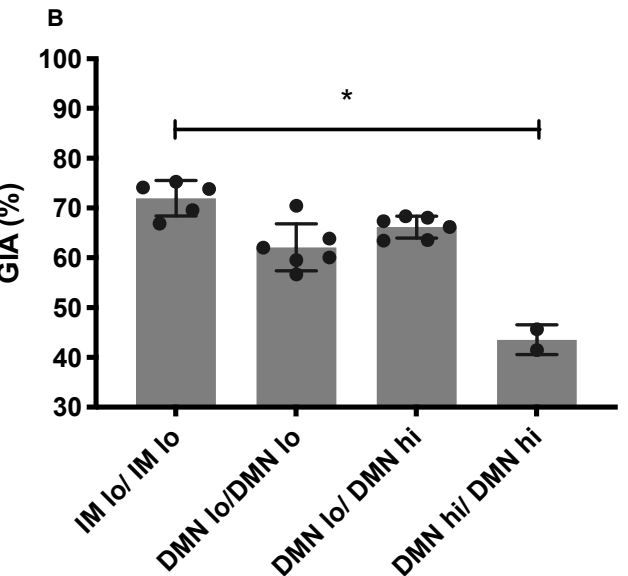

Figure 4. The dose and route of immunization impacts on functional antibody activity against malaria parasites. Female Balb/c mice ( $n=10$ per group) were immunized on day 0 with AdHu5-PfRH5 by the IM route or using DMN patches. The conventional priming and boosting by the IM route, used $1 \times 10^{8}$ ifu and acted as a control regime. Alternatively, mice were administered a low dose of $\mathbf{5} \times \mathbf{1 0 ^ { 4 }}$ ifu by IM injection or DMN patch ("IM lo" or "DMN lo") or a high dose of $\mathbf{5} \times \mathbf{1 0}$ ifu by DMN patch ("DMN hi"). All mice were boosted on day 54 with (A) the control dose of $1 \times 10^{8}$ ifu by the IM route, or $(\mathbf{B})$ with a low or high dose of vaccine by the indicated route. The level of growth inhibitory antibodies to P. falciparum 3D7 clone blood-stage parasites was assessed in purified IgG from serum taken 4 weeks post-boost and pooled into 2 to 6 samples, depending on volumes of available serum. ${ }^{*} p<0.05$, by one-way ANOVA. Bars represent median, error bars represent interquartile range, circles represent individual samples.

In a second study, we examined the functional efficacy induced by repeated low dose immunization and repeated use of DMN patches (Figure $4 \mathrm{~B}$ ). This study confirmed that administration of a low dose of vaccine by the IM or the patch route primed for a high level of growth inhibitory, neutralizing antibodies. Boosting with a low or high vaccine dose with a patch resulted in GIA levels that were equivalent to repeated low vaccine doses by the IM route. In contrast, repeated administration of a high dose using a patch in both immunizations (DMN hi/DMN hi) induced lower levels of GIA.

Overall, these studies demonstrated that repeated administration of a low vaccine dose by either IM or patch route induced substantially higher GIA compared to the conventional repeated high dose $\mathrm{IM}$ regime $\left(\mathrm{IM}, 1 \times 10^{8} \mathrm{ifu}\right)$. Low dose priming with a patch and heterologous boosting, using a high dose by the IM route (Figure $4 \mathrm{~A}$ ), induced the most robust and highest level of parasite growth inhibitory antibodies. This DMN lo/IM 10^8 regimen also induced the highest ratio of antigen-specific to vector-specific antibodies (Figure 2C). Therefore, there is a balance between inducing the highest antigen-specific immunity (and functional antibodies) and using the simplest, homologous immunization regime.

\subsection{Modulation of IgG Subtypes by Altering the Dose and Route of AdHu5-PfRH5}

We examined the antigen-specific IgG1 and IgG2a titres to determine if there were significant differences in the ratio of these subtypes due to the vaccine dose and route of immunization. Only groups that had high levels of GIA were examined and compared to the conventional, high dose repeated IM immunization regimen. It was observed 
that IMlo/IM 10^8 and DMNlo/IM 10^8 significantly increased IgG1 titres compared to repeated high dose IM (Figure 5A). A repeated low dose of vaccine administered with patches (DMNlo/DMNlo) induced significantly reduced antigen-specific IgG2a compared to repeated high dose vaccine by the IM route. Using a patch in the prime skewed the response away from IgG2a; the ratio of IgG1:IgG2a was approximately 100-fold higher when vaccine was administered using a patch in the prime and boost (Figure 5B).

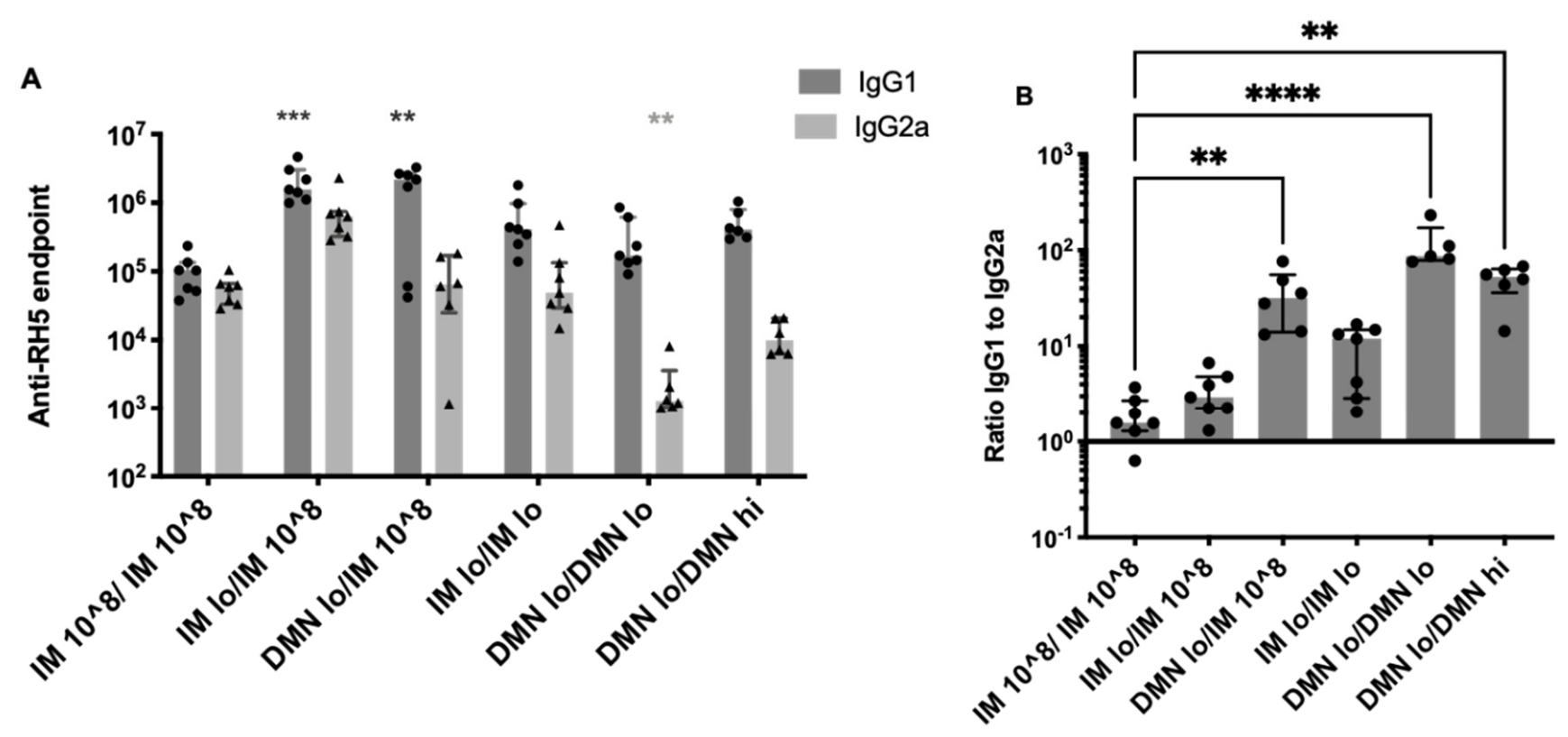

Figure 5. IgG subtypes induced by repeated immunization with low or high dose of vaccine by IM or skin route. Female Balb/c mice ( $n=7$ per group) were immunized on day 0 with AdHu5-PfRH5 by the IM route or using DMN patches. Mice were administered the standard $1 \times 10^{8}$ ifu by IM injection twice ("IM 10^8/IM 10^8"). Alternatively, mice were administered a low dose of $5 \times 10^{4}$ ifu by IM injection or DMN patch ("IM lo" or "DMN lo") or a high dose of $5 \times 10^{7}$ ifu by DMN patch ("DMN hi"). All mice were boosted on day 54 with a low or high dose or $1 \times 10^{8}$ ifu of vaccine by the indicated route. (A) The endpoint titre of RH5-specific IgG1 (dark grey bars) and IgG2a (light grey bars) in serum taken at day 84 (4 weeks post-boost). (B) The ratio of IgG1 to IgG2a in each animal. Bars represent median, lines represent interquartile range, triangles and circles represent individual samples. ${ }^{* *} p<0.01$; ${ }^{* * *} p<0.0005,{ }^{* * * *} p<0.0001$ by Kruskal-Wallis one-way ANOVA comparing all groups to $\mathrm{IM} 10^{\wedge} 8 / \mathrm{IM} 10^{\wedge} 8$.

\subsection{Kinetics of the Antibody Response to the Antigen and to the Vector Induced by the Different Routes and Doses}

Finally, we assessed the kinetics of the IgG response over time after a single immunization with a high $\left(1 \times 10^{8} \mathrm{ifu}\right)$ or low $\left(5 \times 10^{4} \mathrm{ifu}\right)$ dose of vaccine, administered by IM injection or DMN patch (Figure 6). The antibody responses to PfRH5 remained relatively stable in most groups over time; the exceptions being a significant increase in the response in the DMN-lo group at day 56, and a significant decrease in titres in the IM-hi group at day 119 (Figure 6A). Significant increases in anti-AdHu5 IgG were observed in both high dose groups at day 56 compared to earlier time points; the magnitude of this antibody response continued to significantly increase to day 119. Anti-AdHu5 IgG responses could be detected in 1 or 2 mice per group in the low dose groups at day 119. No differences were observed in the kinetics of the antibody responses, to PfRH5 or to AdHu5, when a different route was used to administer a high or low dose of vaccine. 
A

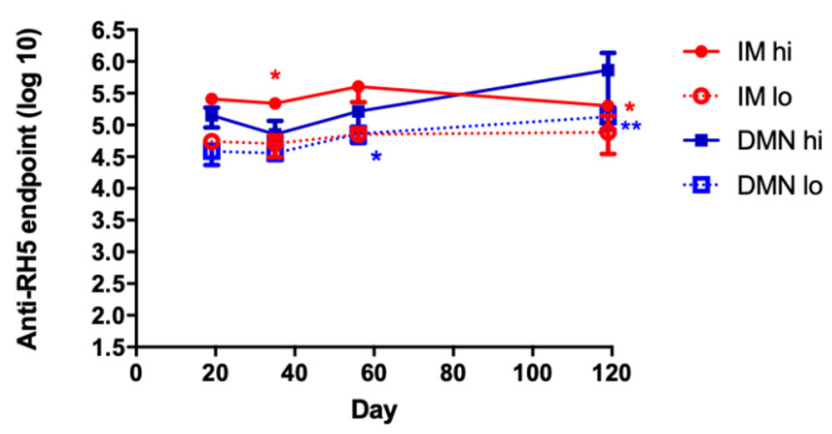

B.

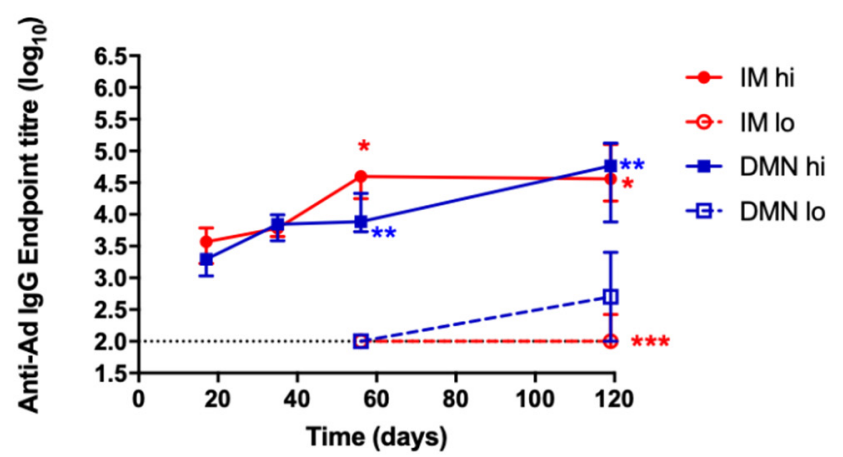

Figure 6. Effect of vaccine dose and route on the kinetics of IgG antibodies to PfRH5 antigen and to the adenovirus vector. Female Balb/c mice were immunized on day 0 with AdHu5-PfRH5 by the IM route or using DMN patches. Mice were administered the standard $1 \times 10^{8}$ ifu by IM injection or with a DMN patch ("IM hi" or "DMN hi"). Alternatively, mice were administered a low dose of $5 \times 10^{4}$ ifu by IM injection or DMN patch ("IM lo" or "DMN lo"). IgG responses to (A) the encoded antigen PfRH5 and (B) to the AdHu5 vector over time ( $n=10$ on day $17, n=7$ on days 35, 56, $n=4$ on d119). Symbols represent median, bars represent interquartile range. For IgG responses to PfRH5, no significant differences in titres were observed over time for IM-lo or DMN-hi; for IM-hi; * $p<0.05$ for day 17 compared to day 35 and day 119 ; $p<0.05$ for day 56 compared to day 119; for DMN-lo, * $p<0.05$; day 17 compared to day 56; * $p<0.05$ day 35 compared to day 119 , ** $p<0.01$; day 17 compared to day 119, by one-way ANOVA, Kruskal-Wallis test with Dunn's multiple comparison. For IgG responses to AdHu5, in IM-hi; * $p<0.05$ for day 17 compared to day 56 and day 119; in DMN-hi ** $p<0.01$ for day 17 compared to day 56 and d119; in IM-lo; ${ }^{* *} p<0.001$ compared to all other timepoints; no significant differences in titre were observed over time for DMN-lo, by one-way ANOVA, Kruskal-Wallis test with Dunn's multiple comparison.

\section{Discussion}

Adenovirus vectored vaccines have become a leading platform technology that is demonstrating potential across multiple infectious disease indications. For COVID-19, clinical efficacy has been demonstrated [41], and a number of adenovirus-based COVID-19 vaccines are now being globally deployed. The ability to repeatedly administer the same adenovirus serotype to significantly increase the anti-transgene immunogenicity would greatly improve immunization programs that utilize this technology. However, the issue of anti-vector immunity [1,42] decreases the potency of adenovirus-based vaccines upon repeated systemic use, and especially within a short timeframe. Several strategies have been developed to prevent or overcome this issue, including the use of a heterologous boost, with for example, an MVA-based vector or a protein subunit boost $[5-9,19,43,44]$. Our study provides a complementary approach of using skin-based delivery with dissolvable microneedle patches to significantly skew the humoral response towards the antigen and away from the vector. We determined that administering lower doses of vaccine skewed the antibody profile towards a higher proportion of antigen-specific, compared to anti-vector, antibodies in mice. Furthermore, stabilizing and administering low doses of vaccine in dissolvable microneedle patches induced the most consistent and favorable skew in the magnitude of antigen-specific IgG, compared to IM injection of liquid vaccine. Finally, immunization with a low vaccine dose with skin patches and boosting with a high dose by the IM route consistently induced the highest level of functional GIA antibodies against the malaria parasite. In contrast, priming with high adenovirus vaccine doses, by either route, induced poor GIA responses.

Dissolvable microneedle technologies are increasingly demonstrating potential as successful vaccine stabilization and delivery systems [45], and have been tested in phase I clinical trials [46-49]. We previously outlined a novel process for producing vaccine-based dissolvable microneedle patches, which does not result in any waste of precious formulated vaccine [15]. We also demonstrated the short-term stability of an adenovirus virus vector in DMN patches [15]. Here, we extended this finding to demonstrate the significant stability advantages of incorporating adenovirus vectored vaccine in these patches. Previous 
attempts to stabilize adenovirus vectors in a solid format have involved drying it onto filter membranes [50] or lyophilization [51]. However, decreases in vaccine viability and potency were observed when adenovirus was dried onto filter paper after 4 weeks at $37^{\circ} \mathrm{C}$ and $45^{\circ} \mathrm{C}$, and secondly, vaccine on these filters or lyophilized still requires reconstitution and the presence of needles and syringes in order to be administered. In contrast, adenovirus vaccine in our DMN patches retained full potency at $40{ }^{\circ} \mathrm{C}$ for somewhere between 8 to 12 weeks, and remained fully viable in this easy-to-administer, needle-free delivery system, at $25^{\circ} \mathrm{C}$ for at least 6 months, when this study completed. A thin film format of adenovirus has recently been described that demonstrated a similar stability to what we observed in DMN patches, however the immunogenicity of an adenovirus vaccine in this film is unknown [52]. This format has similar attributes to our patches with respect to ease of administration. To our knowledge, our study is the first demonstration that a live vaccine can be stabilized to this extent in a microneedle patch format.

We previously examined the induction of immunity by adenovirus-based vaccines delivered with a silicon microneedle technology $[15,35,53,54]$. Other studies have focused on the induction of $\mathrm{T}$ cell responses using long $(800 \mu \mathrm{m})$ dissolvable microneedles fabricated from carboxymethylcellulose (CMC) [24], and only after a single immunization. In a small number of macaques, two adenovirus-based HIV vaccines were coated onto dissolving poly(lactic-co-glycolic acid) (PLGA) microneedles and administered twice, 12 weeks apart. $\mathrm{T}$ cell responses were equivalent in microneedle (MN)-treated and IM injected animals [25]. Humoral responses induced after the primary immunization increased after the second MN immunization, however it is unclear how strong this boosting effect was in comparison to IM delivery. Therefore it is possible to boost weak antigen-specific serum antibody titres in macaques by repeated Ad-DMN immunization, however the comparative magnitude of this effect with injected vaccine is unknown [25]. Our present study suggests that repeated low doses, or a low/high prime/boost, repeated adenovirus regime, could be a more suitable regimen to further increase the magnitude of these responses. We previously demonstrated that these sugar-based microneedles fully dissolve in $<10$ min when administered to porcine skin [15], and therefore deliver vaccine to the body in a short timeframe, similar to the time period for IM delivery. We observed a similar kinetic profile of the antibody response after a single immunization using an IM injection or a DMN patch (Figure 6). Therefore, although the magnitude of the antibody responses were dependent on the dose and delivery system, the kinetic profile of the serum antibody responses were independent of the vaccine format and route.

Clinical trials have assessed the induction of immune responses by repeated adenovirus vector based vaccine administration. For example, repeated IM use of an adenovirus type 35 by the IM route [18] demonstrated that repeated high dose immunization induced higher CD8+ T cells to the encoded antigens, however the phenotype of the boosted CD8+ $\mathrm{T}$ cell response was less polyfunctional compared to a single immunization. This suggests that repeated higher doses may not be as effective at inducing protective CD8+ T cells. In malaria vaccine trials, a second immunization with two AdHu5-based vaccines, each at $1 \times 10^{10}$ virus particles, administered concurrently, did not boost antibody responses [55]. In a phase I clinical trial of heterologous prime-boost regime for an Ebola virus vaccine, administering the lowest dose of a chimpanzee adenovirus (ChAd3, $\left.1 \times 10^{10} \mathrm{vp}\right)$ primed for a substantially stronger IgG and neutralizing antibody response subsequent to an MVA boost, compared to priming with $2.5 \times 10^{10} \mathrm{vp}$ or $5 \times 10^{10} \mathrm{vp}$ of ChAd3, although the low number of volunteers in each group prevents statistical analysis [6]. Repeated use of an adenovirus-based COVID-19 vaccine, namely ChAdOx1-nCoV-19 [56], or malaria vaccine, namely ChAd3-METRAP [57], did not boot $\mathrm{T}$ cell responses in either study, but was demonstrated to be capable of increasing antibody titre and modulating antibody effector function to the spike antigen of SARS-CoV2 [56], but not to the malaria antigen [58]; however robust statistical analyses was not feasible in the former study. It is well recognized that $\mathrm{T}$ cell receptor (TCR) stimulation strength affects the phenotype of $\mathrm{T}$ cell responses; including the generation and function of follicular helper $\mathrm{T}$ cells, which are 
critical to modulating the induction of $\mathrm{B}$ cell responses [57]. High antigen dose stimulates $\mathrm{T}$ cells of both high and low avidity, thereby permitting expansion of $\mathrm{T}$ cells with lower functional quality [57]. Furthermore, increasing antigen dose results in higher expression on T cells of inhibitory receptors, such as PD-1 and CTLA-4 [59]. Here, we add further to these data by demonstrating that a low, compared to high, adenovirus vaccine dose resulted in a significant skew in antibody responses towards the encoded transgene and away from the vector. Decreasing the dose of total adenovirus vector proteins at The effect of the TCR stimulation strength on T cell responses is likely one component of why lower doses of vaccine were more immunogenic in our study, and potentially leads to better priming in the clinicthe time of administration also likely reduces the opportunity for $\mathrm{B}$ and $\mathrm{T}$ cells to be exposed to vector antigens, while transgene antigen production is maintained above a critical threshold. Finally, decreasing the dose likely also reduced the induction of innate immune response genes, which have been shown to inversely correlate with antigen expression from adenovirus vectored vaccines [60].

\section{Conclusions}

Our findings demonstrate that priming with a low dose of adenovirus vaccine using a skin patch and performing a high dose IM boost with the same vaccine induces the highest level of functional, parasite growth inhibitory antibodies and low anti-vector responses. Fast, cost-effective deployment of vaccines is a required attribute in a vaccine-based response to disease outbreaks. Distribution in regular, room temperature drug distribution networks would address the current cold chain constraints in vaccine deployment. We have developed a stabilized vaccine platform that addresses this issue. Secondly, our skin patch system also addresses the constraints surrounding training vaccinators to prepare and inject vaccines, and safely dispose of biohazardous waste. In addition to these solutions to vaccine access issues, we demonstrate here that this patch system is also capable of significantly enhancing the induction of protective immune responses compared to conventional injection-based immunization. We conclude that further translation of this technology is warranted based on these findings and on the clinical need to address these access and efficacy issues.

Supplementary Materials: The following are available online at https://www.mdpi.com/2076-393 X/9/3/299/s1, Figure S1: Graphical representation of immunization regimes and schedules used in this study.

Author Contributions: O.F., A.C.M. and S.J.D. designed the experiments; O.F., A.C.M. and S.J.D. wrote the manuscript; O.F., K.D., prepared the microneedle patches; O.F., K.D. and J.S.L. conducted immunological assays; J.M.M. conducted GIA assays; J.J. prepared the RH5.1 protein for ELISAs; S.E.S. conducted pre-GIA ELISAs; O.F., A.C.M. and S.J.D. analyzed the data. All authors have read and agreed to the published version of the manuscript.

Funding: This work was supported by Health Research Board, Ireland, Patient Oriented Research grant POR/2012/95. S.J.D. is a Jenner Investigator, a Lister Institute Research Prize Fellow and a Wellcome Trust Senior Fellow [106917/Z/15/Z]; for the purpose of Open Access, the author has applied a CC BY public copyright licence to any Author Accepted Manuscript version arising from this submission.

Institutional Review Board Statement: The study was conducted in accordance with the terms of licenses from the Irish Department of Health and Children under the Cruelty to Animals Act (license number B100/4034) and according to the approval of the UCC AECC Committee.

Informed Consent Statement: Not applicable.

Data Availability Statement: The data presented in this study are openly available in FigShare at https://doi.org/10.6084/m9.figshare.14198867.v1. 
Acknowledgments: The authors would like to thank Conor O'Mahony, Tyndall National Institute, UCC for supplying PDMS moulds. Geneviève Labbé and the Viral Vector Core Facility, Jenner Institute, University of Oxford for assistance and the staff of the Biological Services Unit, UCC. This work was supported by Health Research Board, Ireland, Patient Oriented Research grant POR/2012/95. S.J.D. is a Jenner Investigator, a Lister Institute Research Prize Fellow and a Wellcome Trust Senior Fellow [106917/Z/15/Z]; for the purpose of Open Access, the author has applied a CC BY public copyright licence to any Author Accepted Manuscript version arising from this submission.

Conflicts of Interest: O.F. and A.C.M. are inventors of patents that have been or may be licensed to companies developing microneedle-based products. This potential competing interest has been disclosed and is being managed by University College Cork. S.J.D. is a named inventor on patent applications relating to $\mathrm{RH} 5$ and/or adenovirus-based vaccines and immunization regimens. All other authors declare that they have no known competing financial interests. None of the authors have personal relationships that could have appeared to influence the work reported in this paper.

\section{References}

1. Zhu, F.C.; Guan, X.H.; Li, Y.H.; Huang, J.Y.; Jiang, T.; Hou, L.H.; Li, J.X.; Yang, B.F.; Wang, L.; Wang, W.J.; et al. Immunogenicity and safety of a recombinant adenovirus type-5-vectored COVID-19 vaccine in healthy adults aged 18 years or older: A randomised, double-blind, placebo-controlled, phase 2 trial. Lancet 2020, 396, 479-488. [CrossRef]

2. Mercado, N.B.; Zahn, R.; Wegmann, F.; Loos, C.; Chandrashekar, A.; Yu, J.; Liu, J.; Peter, L.; McMahan, K.; Tostanoski, L.H.; et al. Single-shot Ad26 vaccine protects against SARS-CoV-2 in rhesus macaques. Nature 2020, 586, 583-588. [CrossRef] [PubMed]

3. Ramasamy, M.N.; Minassian, A.M.; Ewer, K.J.; Flaxman, A.L.; Folegatti, P.M.; Owens, D.R.; Voysey, M.; Aley, P.K.; Angus, B.; Babbage, G.; et al. Safety and immunogenicity of ChAdOx1 nCoV-19 vaccine administered in a prime-boost regimen in young and old adults (COV002): A single-blind, randomised, controlled, phase 2/3 trial. Lancet 2020, 396, 1979-1993. [CrossRef]

4. Wolfson, L.J.; Gasse, F.; Lee-Martin, S.P.; Lydon, P.; Magan, A.; Tibouti, A.; Johns, B.; Hutubessy, R.; Salama, P.; Okwo-Bele, J.M. Estimating the costs of achieving the WHO-UNICEF Global Immunization Vision and Strategy, 2006-2015. Bull. World Health Organ. 2008, 86, 27-39. [CrossRef] [PubMed]

5. Payne, R.O.; Silk, S.E.; Elias, S.C.; Miura, K.; Diouf, A.; Galaway, F.; de Graaf, H.; Brendish, N.J.; Poulton, I.D.; Griffiths, O.J.; et al. Human vaccination against RH5 induces neutralizing antimalarial antibodies that inhibit RH5 invasion complex interactions. JCI Insight 2017, 2, e96381. [CrossRef] [PubMed]

6. Ewer, K.; Rampling, T.; Venkatraman, N.; Bowyer, G.; Wright, D.; Lambe, T.; Imoukhuede, E.B.; Payne, R.; Fehling, S.K.; Strecker, T.; et al. A Monovalent Chimpanzee Adenovirus Ebola Vaccine Boosted with MVA. N. Engl. J. Med. 2016, 374, 1635-1646. [CrossRef] [PubMed]

7. Sheehy, S.H.; Duncan, C.J.; Elias, S.C.; Collins, K.A.; Ewer, K.J.; Spencer, A.J.; Williams, A.R.; Halstead, F.D.; Moretz, S.E.; Miura, K.; et al. Phase Ia clinical evaluation of the Plasmodium falciparum blood-stage antigen MSP1 in ChAd63 and MVA vaccine vectors. Mol. Ther. 2011, 19, 2269-2276. [CrossRef]

8. Milligan, I.D.; Gibani, M.M.; Sewell, R.; Clutterbuck, E.A.; Campbell, D.; Plested, E.; Nuthall, E.; Voysey, M.; Silva-Reyes, L.; McElrath, M.J.; et al. Safety and Immunogenicity of Novel Adenovirus Type 26- and Modified Vaccinia Ankara-Vectored Ebola Vaccines: A Randomized Clinical Trial. JAMA 2016, 315, 1610-1623. [CrossRef]

9. Hodgson, S.H.; Choudhary, P.; Elias, S.C.; Milne, K.H.; Rampling, T.W.; Biswas, S.; Poulton, I.D.; Miura, K.; Douglas, A.D.; Alanine, D.G.; et al. Combining viral vectored and protein-in-adjuvant vaccines against the blood-stage malaria antigen AMA1: Report on a phase 1a clinical trial. Mol. Ther. 2014, 22, 2142-2154. [CrossRef]

10. Barnes, E.; Folgori, A.; Capone, S.; Swadling, L.; Aston, S.; Kurioka, A.; Meyer, J.; Huddart, R.; Smith, K.; Townsend, R.; et al. Novel adenovirus-based vaccines induce broad and sustained T cell responses to HCV in man. Sci. Transl. Med. 2012, 4, $115 \mathrm{ra111.}$ [CrossRef]

11. Baden, L.R.; Karita, E.; Mutua, G.; Bekker, L.G.; Gray, G.; Page-Shipp, L.; Walsh, S.R.; Nyombayire, J.; Anzala, O.; Roux, S.; et al. Assessment of the Safety and Immunogenicity of 2 Novel Vaccine Platforms for HIV-1 Prevention: A Randomized Trial. Ann. Intern. Med. 2016, 164, 313-322. [CrossRef]

12. Carey, J.B.; Vrdoljak, A.; O’Mahony, C.; Hill, A.V.; Draper, S.J.; Moore, A.C. Microneedle-mediated immunization of an adenovirusbased malaria vaccine enhances antigen-specific antibody immunity and reduces anti-vector responses compared to the intradermal route. Sci. Rep. 2014, 4, 6154. [CrossRef] [PubMed]

13. Allen, E.A.; O'Mahony, C.; Cronin, M.; O’Mahony, T.; Moore, A.C.; Crean, A.M. Dissolvable microneedle fabrication using piezoelectric dispensing technology. Int. J. Pharm. 2016, 500, 1-10. [CrossRef] [PubMed]

14. Moore, A.; Vrdoljak, A. Method for Fabricating a Microneedle, and Produced Microneedle. Patent WO2012153266 A3, 6 June 2012.

15. Vrdoljak, A.; Allen, E.A.; Ferrara, F.; Temperton, N.J.; Crean, A.M.; Moore, A.C. Induction of broad immunity by thermostabilised vaccines incorporated in dissolvable microneedles using novel fabrication methods. J. Control Release 2016, 225, 192-204. [CrossRef] 
16. Donadei, A.; Flynn, O.; Moore, A.C. Composition and Method for Stabilising Vaccines in a Solid Dosage Format. Patent WO/2019/238779, 19 December 2019.

17. Skeiky, Y.A.; Sadoff, J.C. Advances in tuberculosis vaccine strategies. Nat. Rev. Microbiol. 2006, 4, 469-476. [CrossRef] [PubMed]

18. van Zyl-Smit, R.N.; Esmail, A.; Bateman, M.E.; Dawson, R.; Goldin, J.; van Rikxoort, E.; Douoguih, M.; Pau, M.G.; Sadoff, J.C.; McClain, J.B.; et al. Safety and Immunogenicity of Adenovirus 35 Tuberculosis Vaccine Candidate in Adults with Active or Previous Tuberculosis. A Randomized Trial. Am. J. Respir. Crit. Care Med. 2017, 195, 1171-1180. [CrossRef]

19. Zhu, F.C.; Wurie, A.H.; Hou, L.H.; Liang, Q.; Li, Y.H.; Russell, J.B.; Wu, S.P.; Li, J.X.; Hu, Y.M.; Guo, Q.; et al. Safety and immunogenicity of a recombinant adenovirus type- 5 vector-based Ebola vaccine in healthy adults in Sierra Leone: A single-centre, randomised, double-blind, placebo-controlled, phase 2 trial. Lancet 2017, 389, 621-628. [CrossRef]

20. Dolzhikova, I.V.; Zubkova, O.V.; Tukhvatulin, A.I.; Dzharullaeva, A.S.; Tukhvatulina, N.M.; Shcheblyakov, D.V.; Shmarov, M.M.; Tokarskaya, E.A.; Simakova, Y.V.; Egorova, D.A.; et al. Safety and immunogenicity of GamEvac-Combi, a heterologous VSV- and Ad5-vectored Ebola vaccine: An open phase I/II trial in healthy adults in Russia. Hum. Vaccines Immunother. 2017, 13, 613-620. [CrossRef]

21. Folegatti, P.M.; Bittaye, M.; Flaxman, A.; Lopez, F.R.; Bellamy, D.; Kupke, A.; Mair, C.; Makinson, R.; Sheridan, J.; Rohde, C.; et al. Safety and immunogenicity of a candidate Middle East respiratory syndrome coronavirus viral-vectored vaccine: A doseescalation, open-label, non-randomised, uncontrolled, phase 1 trial. Lancet Infect. Dis. 2020, 20, 816-826. [CrossRef]

22. Sridhar, S.; Reyes-Sandoval, A.; Draper, S.J.; Moore, A.C.; Gilbert, S.C.; Gao, G.P.; Wilson, J.M.; Hill, A.V. Single-dose protection against Plasmodium berghei by a simian adenovirus vector using a human cytomegalovirus promoter containing intron $\mathrm{A}$. J. Virol. 2008, 82, 3822-3833. [CrossRef]

23. Reyes-Sandoval, A.; Sridhar, S.; Berthoud, T.; Moore, A.C.; Harty, J.T.; Gilbert, S.C.; Gao, G.; Ertl, H.C.J.; Wilson, J.C.; Hill, A.V.S. Single-dose immunogenicity and protective efficacy of simian adenoviral vectors against Plasmodium berghei. Eur. J. Immunol. 2008, 38, 732-741. [CrossRef]

24. Bachy, V.; Hervouet, C.; Becker, P.D.; Chorro, L.; Carlin, L.M.; Herath, S.; Papagatsias, T.; Barbaroux, J.B.; Oh, S.J.; Benlahrech, A.; et al. Langerin negative dendritic cells promote potent CD8+ T-cell priming by skin delivery of live adenovirus vaccine microneedle arrays. Proc. Natl. Acad. Sci. USA 2013, 110, 3041-3046. [CrossRef] [PubMed]

25. DeMuth, P.C.; Li, A.V.; Abbink, P.; Liu, J.; Li, H.; Stanley, K.A.; Smith, K.M.; Lavine, C.L.; Seaman, M.S.; Kramer, J.A.; et al. Vaccine delivery with microneedle skin patches in nonhuman primates. Nat. Biotechnol. 2013, 31, 1082-1085. [CrossRef]

26. Draper, S.J.; Sack, B.K.; King, C.R.; Nielsen, C.M.; Rayner, J.C.; Higgins, M.K.; Long, C.A.; Seder, R.A. Malaria Vaccines: Recent Advances and New Horizons. Cell Host Microbe 2018, 24, 43-56. [CrossRef] [PubMed]

27. Douglas, A.D.; Baldeviano, G.C.; Lucas, C.M.; Lugo-Roman, L.A.; Crosnier, C.; Bartholdson, S.J.; Diouf, A.; Miura, K.; Lambert, L.E.; Ventocilla, J.A.; et al. A PfRH5-based vaccine is efficacious against heterologous strain blood-stage Plasmodium falciparum infection in aotus monkeys. Cell Host Microbe 2015, 17, 130-139. [CrossRef] [PubMed]

28. Mahdi Abdel Hamid, M.; Remarque, E.J.; van Duivenvoorde, L.M.; van der Werff, N.; Walraven, V.; Faber, B.W.; Kocken, C.H.; Thomas, A.W. Vaccination with Plasmodium knowlesi AMA1 formulated in the novel adjuvant co-vaccine HT protects against blood-stage challenge in rhesus macaques. PLoS ONE 2011, 6, e20547. [CrossRef] [PubMed]

29. Singh, S.; Miura, K.; Zhou, H.; Muratova, O.; Keegan, B.; Miles, A.; Martin, L.B.; Saul, A.J.; Miller, L.H.; Long, C.A. Immunity to recombinant plasmodium falciparum merozoite surface protein 1 (MSP1): Protection in Aotus nancymai monkeys strongly correlates with anti-MSP1 antibody titer and in vitro parasite-inhibitory activity. Infect. Immun. 2006, 74, 4573-4580. [CrossRef]

30. Douglas, A.D.; Williams, A.R.; Illingworth, J.J.; Kamuyu, G.; Biswas, S.; Goodman, A.L.; Wyllie, D.H.; Crosnier, C.; Miura, K.; Wright, G.J.; et al. The blood-stage malaria antigen PfRH5 is susceptible to vaccine-inducible cross-strain neutralizing antibody. Nat. Commun. 2011, 2, 601. [CrossRef]

31. Donadei, A.; Kraan, H.; Ophorst, O.; Flynn, O.; O’Mahony, C.; Soema, P.C.; Moore, A.C. Skin delivery of trivalent Sabin inactivated poliovirus vaccine using dissolvable microneedle patches induces neutralizing antibodies. J. Control Release 2019, 311, 96-103. [CrossRef]

32. Lanza, J.S.; Vucen, S.; Flynn, O.; Donadei, A.; Cojean, S.; Loiseau, P.M.; Fernandes, A.; Frezard, F.; Moore, A.C. A TLR9-adjuvanted vaccine formulated into dissolvable microneedle patches or cationic liposomes protects against leishmaniasis after skin or subcutaneous immunization. Int. J. Pharm. 2020, 586, 119390. [CrossRef]

33. EMEA. ICH Topic Q1 A (R2). Stability Testing of New Drug Substances and Products. 2006. Available online: https://www.ema. europa.eu/en/documents/scientific-guideline/ich-q-1-r2-stability-testing-new-drug-substances-products-step-5_en.pdf (accessed on 1 January 2021).

34. Bernelin-Cottet, C.; Urien, C.; McCaffrey, J.; Collins, D.; Donadei, A.; McDaid, D.; Jakob, V.; Barnier-Quer, C.; Collin, N.; Bouguyon, E.; et al. Electroporation of a nanoparticle-associated DNA vaccine induces higher inflammation and immunity compared to its delivery with microneedle patches in pigs. J. Control Release 2019, 308, 14-28. [CrossRef]

35. Vrdoljak, A.; McGrath, M.G.; Carey, J.B.; Draper, S.J.; Hill, A.V.; O’Mahony, C.; Crean, A.M.; Moore, A.C. Coated microneedle arrays for transcutaneous delivery of live virus vaccines. J. Control Release 2012, 159, 34-42. [CrossRef] 
36. Draper, S.J.; Moore, A.C.; Goodman, A.L.; Long, C.A.; Holder, A.A.; Gilbert, S.C.; Hill, F.; Hill, A.V. Effective induction of high-titer antibodies by viral vector vaccines. Nat. Med. 2008, 14, 819-821. [CrossRef]

37. Jin, J.; Tarrant, R.D.; Bolam, E.J.; Angell-Manning, P.; Soegaard, M.; Pattinson, D.J.; Dulal, P.; Silk, S.E.; Marshall, J.M.; Dabbs, R.A.; et al. Production, quality control, stability, and potency of cGMP-produced Plasmodium falciparum RH5.1 protein vaccine expressed in Drosophila S2 cells. NPJ Vaccines 2018, 3, 32. [CrossRef] [PubMed]

38. Alanine, D.G.W.; Quinkert, D.; Kumarasingha, R.; Mehmood, S.; Donnellan, F.R.; Minkah, N.K.; Dadonaite, B.; Diouf, A.; Galaway, F.; Silk, S.E.; et al. Human Antibodies that Slow Erythrocyte Invasion Potentiate Malaria-Neutralizing Antibodies. Cell 2019, 178, 216-228.e221. [CrossRef]

39. Malkin, E.M.; Diemert, D.J.; McArthur, J.H.; Perreault, J.R.; Miles, A.P.; Giersing, B.K.; Mullen, G.E.; Orcutt, A.; Muratova, O.; Awkal, M.; et al. Phase 1 clinical trial of apical membrane antigen 1: An asexual blood-stage vaccine for Plasmodium falciparum malaria. Infect. Immun. 2005, 73, 3677-3685. [CrossRef] [PubMed]

40. de Cassan, S.C.; Shakri, A.R.; Llewellyn, D.; Elias, S.C.; Cho, J.S.; Goodman, A.L.; Jin, J.; Douglas, A.D.; Suwanarusk, R.; Nosten, F.H.; et al. Preclinical Assessment of Viral Vectored and Protein Vaccines Targeting the Duffy-Binding Protein Region II of Plasmodium Vivax. Front. Immunol. 2015, 6, 348. [CrossRef]

41. Voysey, M.; Clemens, S.A.C.; Madhi, S.A.; Weckx, L.Y.; Folegatti, P.M.; Aley, P.K.; Angus, B.; Baillie, V.L.; Barnabas, S.L.; Bhorat, Q.E.; et al. Safety and efficacy of the ChAdOx1 nCoV-19 vaccine (AZD1222) against SARS-CoV-2: An interim analysis of four randomised controlled trials in Brazil, South Africa, and the UK. Lancet 2021, 397, 99-111. [CrossRef]

42. Priddy, F.H.; Brown, D.; Kublin, J.; Monahan, K.; Wright, D.P.; Lalezari, J.; Santiago, S.; Marmor, M.; Lally, M.; Novak, R.M.; et al. Safety and immunogenicity of a replication-incompetent adenovirus type $5 \mathrm{HIV}-1$ clade B gag/pol/nef vaccine in healthy adults. Clin. Infect. Dis. 2008, 46, 1769-1781. [CrossRef] [PubMed]

43. Callendret, B.; Vellinga, J.; Wunderlich, K.; Rodriguez, A.; Steigerwald, R.; Dirmeier, U.; Cheminay, C.; Volkmann, A.; Brasel, T.; Carrion, R.; et al. A prophylactic multivalent vaccine against different filovirus species is immunogenic and provides protection from lethal infections with Ebolavirus and Marburgvirus species in non-human primates. PLoS ONE 2018, 13, e0192312.

44. Salisch, N.C.; Izquierdo Gil, A.; Czapska-Casey, D.N.; Vorthoren, L.; Serroyen, J.; Tolboom, J.; Saeland, E.; Schuitemaker, H.; Zahn, R.C. Adenovectors encoding RSV-F protein induce durable and mucosal immunity in macaques after two intramuscular administrations. NPJ Vaccines 2019, 4, 54. [CrossRef] [PubMed]

45. Marshall, S.; Sahm, L.J.; Moore, A.C. The success of microneedle-mediated vaccine delivery into skin. Hum. Vaccines Immunother. 2016, 12, 2975-2983. [CrossRef] [PubMed]

46. Rouphael, N.G.; Paine, M.; Mosley, R.; Henry, S.; McAllister, D.V.; Kalluri, H.; Pewin, W.; Frew, P.M.; Yu, T.; Thornburg, N.J.; et al. The safety, immunogenicity, and acceptability of inactivated influenza vaccine delivered by microneedle patch (TIV-MNP 2015): A randomised, partly blinded, placebo-controlled, phase 1 trial. Lancet 2017, 390, 649-658. [CrossRef]

47. Hirobe, S.; Azukizawa, H.; Hanafusa, T.; Matsuo, K.; Quan, Y.S.; Kamiyama, F.; Katayama, I.; Okada, N.; Nakagawa, S. Clinical study and stability assessment of a novel transcutaneous influenza vaccination using a dissolving microneedle patch. Biomaterials 2015, 57, 50-58. [CrossRef] [PubMed]

48. Hirobe, S.; Matsuo, K.; Quan, Y.S.; Kamiyama, F.; Morito, H.; Asada, H.; Takaya, Y.; Mukai, Y.; Okada, N.; Nakagawa, S. Clinical study of transcutaneous vaccination using a hydrogel patch for tetanus and diphtheria. Vaccine 2012, 30, 1847-1854. [CrossRef]

49. Norman, J.J.; Arya, J.M.; McClain, M.A.; Frew, P.M.; Meltzer, M.I.; Prausnitz, M.R. Microneedle patches: Usability and acceptability for self-vaccination against influenza. Vaccine 2014, 32, 1856-1862. [CrossRef]

50. Alcock, R.; Cottingham, M.G.; Rollier, C.S.; Furze, J.; De Costa, S.D.; Hanlon, M.; Spencer, A.J.; Honeycutt, J.D.; Wyllie, D.H.; Gilbert, S.C.; et al. Long-term thermostabilization of live poxviral and adenoviral vaccine vectors at supraphysiological temperatures in carbohydrate glass. Sci. Transl. Med. 2010, 2, 19ra12. [CrossRef]

51. Croyle, M.A.; Roessler, B.J.; Davidson, B.L.; Hilfinger, J.M.; Amidon, G.L. Factors that influence stability of recombinant adenoviral preparations for human gene therapy. Pharm. Dev. Technol. 1998, 3, 373-383. [CrossRef]

52. Bajrovic, I.; Schafer, S.C.; Romanovicz, D.K.; Croyle, M.A. Novel technology for storage and distribution of live vaccines and other biological medicines at ambient temperature. Sci. Adv. 2020,6, eaau4819. [CrossRef]

53. Carey, J.B.; Pearson, F.E.; Vrdoljak, A.; McGrath, M.G.; Crean, A.M.; Walsh, P.T.; Doody, T.; O'Mahony, C.; Hill, A.V.S.; Moore, A.C. Microneedle Array Design Determines the Induction of Protective Memory CD8(+) T Cell Responses Induced by a Recombinant Live Malaria Vaccine in Mice. PLoS ONE 2011, 6, e22442. [CrossRef] [PubMed]

54. Pearson, F.E.; O'Mahony, C.; Moore, A.C.; Hill, A.V. Induction of CD8(+) T cell responses and protective efficacy following microneedle-mediated delivery of a live adenovirus-vectored malaria vaccine. Vaccine 2015, 33, 3248-3255. [CrossRef]

55. Tamminga, C.; Sedegah, M.; Regis, D.; Chuang, I.; Epstein, J.E.; Spring, M.; Mendoza-Silveiras, J.; McGrath, S.; Maiolatesi, S.; Reyes, S.; et al. Adenovirus-5-vectored P. falciparum vaccine expressing CSP and AMA1. Part B: Safety, immunogenicity and protective efficacy of the CSP component. PLOS ONE 2011, 6, e25868. [CrossRef] [PubMed]

56. Barrett, J.R.; Belij-Rammerstorfer, S.; Dold, C.; Ewer, K.J.; Folegatti, P.M.; Gilbride, C.; Halkerston, R.; Hill, J.; Jenkin, D.; Stockdale, L.; et al. Phase 1/2 trial of SARS-CoV-2 vaccine ChAdOx1 nCoV-19 with a booster dose induces multifunctional antibody responses. Nat. Med. 2020, 27, 279-288. [CrossRef] [PubMed]

57. Fazilleau, N.; McHeyzer-Williams, L.J.; Rosen, H.; McHeyzer-Williams, M.G. The function of follicular helper T cells is regulated by the strength of $\mathrm{T}$ cell antigen receptor binding. Nat. Immunol. 2009, 10, 375-384. [CrossRef] [PubMed] 
58. Bliss, C.M.; Bowyer, G.; Anagnostou, N.A.; Havelock, T.; Snudden, C.M.; Davies, H.; de Cassan, S.C.; Grobbelaar, A.; Lawrie, A.M.; Venkatraman, N.; et al. Assessment of novel vaccination regimens using viral vectored liver stage malaria vaccines encoding ME-TRAP. Sci. Rep. 2018, 8, 3390. [CrossRef]

59. Billeskov, R.; Wang, Y.; Solaymani-Mohammadi, S.; Frey, B.; Kulkarni, S.; Andersen, P.; Agger, E.M.; Sui, Y.; Berzofsky, J.A. Low Antigen Dose in Adjuvant-Based Vaccination Selectively Induces CD4 T Cells with Enhanced Functional Avidity and Protective Efficacy. J. Immunol. 2017, 198, 3494-3506. [CrossRef]

60. Quinn, K.M.; Zak, D.E.; Costa, A.; Yamamoto, A.; Kastenmuller, K.; Hill, B.J.; Lynn, G.M.; Darrah, P.A.; Lindsay, R.W.; Wang, L.; et al. Antigen expression determines adenoviral vaccine potency independent of IFN and STING signaling. J. Clin. Investig. 2015, 125, 1129-1146. [CrossRef] 